auch für das betroffene Management. Wer bereits das Vorgängermodell CMM kennt, findet alle Änderungen in übersichtlicher Form zusammengefasst. Aufgrund seiner klaren Struktur (die auch von einem zusätzlich erhältlichen Poster unterstützt wird) eignet sich das Buch ebenfalls als Begleitbuch zu entsprechenden Lehrveranstaltungen.

In neun Kapiteln und umfangreichen Anhängen, die unter anderem eine Übersetzung wesentlicher Teile des CMMI umfassen, werden Entstehung, Aufbau, Einführungs- und Anwendungsaspekte beschrieben. Eine Nutzenbetrachtung und kritische Bewertung, eine Auseinandersetzung mit oftmals vorgebrachten typischen Kritikpunkten sowie eine Darstellung der CMMI-Appraisals/Assessments runden das Buch ab. Darüber hinaus werden die mit CMMI vergleichbaren oder sinnvoll in Bezug zu setzenden wichtigen Modelle und Methoden (beispielsweise ISO 9001, SPICE, ITIL, V-Modell XT, agile Prozesse/XP) im CMMI-Kontext bewertend ein- und zugeordnet.

Es wird rasch deutlich, dass CMMI und die darin abgebildeten Ursache-WirkungsZusammenhänge komplex sind. Ralf Kneuper entwirrt hier strukturiert, in genau der richtigen Ausführlichkeit und anhand begleitender Beispiele, unterstützender Graphiken und praktischer Hinweise. Wichtiges und weniger Zentrales im Modell werden getrennt und in eine Reihenfolge gebracht, um den Überblick und das Herangehen an eine spätere Anwendung von CMMI zu erleichtern. Die Lektüre des Buches liefert zudem die Begründung, warum Akzeptanz und Erfolg von CMMI-Projekten auch in hohem Maße von der Qualifikation und Kompetenz hinzugezogener Berater abhängt. Und schließlich wird verständlich, dass man, um mit CMMI erfolgreich sein zu können, lernen muss, „neben sich treten“ $\mathrm{zu}$ können und eine gewisse Beharrlichkeit zu zeigen. Denn der für den CMMI-Einsatz notwendige „Kulturwandel“ vollzieht sich meist nur mühsam.

In der 3. Auflage würden an einigen Stellen zusätzliche Abbildungen das Verständni noch weiter erleichtern. Vielleicht gelingt es zudem, Beispiele hinzuzunehmen oder vor handene Erfahrungsberichte auszubauen. Doch unabhängig von immer denkbaren Verbesserungen schließt der Leser die sorgfältige Lektüre dieses auch typographisch sehr ansprechenden Buches wohl informiert ab. Er weiß jetzt, was hinter CMMI steht, worauf es dort ankommt und kann eigene Erfahrungen sammeln oder ergänzen.

Matthias Knoll, Darmstadt

\section{Heisig, P.; Mertins K. (Hrsg.) \\ Integration von Wissensmanagement in Geschäftsprozesse \\ ISBN 3-000-17244-0, EuReKI,}

Berlin 2005, 205 Seiten, $€ 53,00$

Die Bedeutung von Wissen als zentralem Produktionsfaktor für den Standort Deutschland ist hinlänglich bekannt, ebenso wie die Notwendigkeit für Unternehmen, ihre Geschäftsprozesse entlang der Wertschöpfungskette zu optimieren. Dafür stehen eine Vielzahl von Methoden und Werkzeugen zur Verfügung. Umso verwunderlicher ist es, dass der Faktor Wissen bisher kaum in diesem Optimierungsprozess Einzug gehalten hat, bzw. sich in den verwendeten Methoden nicht widerspiegelt.

Der Autor nimmt sich mit seiner Dissertation eben dieses Problems an und stellt eine Methodik zur Einbeziehung des Wissensmanagements (WM) in Geschäftsprozesse vor. Es gliedert sich in sieben Kapitel mit insgesamt 205 Seiten. Nach einer kurzen Einleitung werden im zweiten Kapitel die verwendeten Begriffe Wissen, Geschäftsprozess und Wissensmanagement definiert. Das dritte Kapitel erklärt die Anforderungen, die der Autor an eine Methode des integrierten Wissensmanagements stellt, welche auf einer Befragung von WM-Experten beruhen. Im vierten Kapitel werden bisherige Ansätze zum prozessorientierten WM vorgestellt und bewertet, bevor der Autor im fünften Kapitel seine Methodik (GPO-WM) detailliert vorstellt. Das sechste Kapitel stellt zwei Fallstudien vor, bei denen diese Vorgehensweise praktisch durchführt wurde. In Kapitel sieben wird abschließend eine Zusammenfassung dargestellt.

Die in dem Buch herausgearbeiteten Anforderungen an eine solche Methodik (u. a. Ganzheitlichkeit, Ausgewogenheit, Beteiligungsorientierung) dienen als Maßstab der Analyse bestehender Verfahren. Hauptkritikpunkt des Autors ist dabei die mangelnde Einbindung der Mitarbeiter bei den einzelnen Verfahren, d. h. kein beteiligungsorientiertes Vorgehen, sowie die fehlende theoretische und empirische Grundlage. Aufbauend auf dieser Analyse wird eine Methode (GPO-WM) vorgestellt und basierend auf den Ergebnissen o. a. Expertenbefragung ein Referenzmodell mit drei Ebenen definiert: Geschäftsprozesse, WM-Kernaktivitäten (Wissen erzeugen, speichern, verteilen und anwenden) und Gestaltungsfelder (Personalmanagement, Controlling etc.). Das daraus resultierende Vorgehensmodell beinhaltet die Phasen WM-Strategie, -Lösung und -Einführung, das in insgesamt neun Schritten von der Definition der Strategie zur Umsetzung und Nutzung vorangeht. Weiterhin beschreibt der Autor die Analysemodule
WM-Assessment, Prozessanalyse und Lösungsdatenbank. Das Assessment beruht größtenteils auf der Befragung der Mitarbeiter bzw. Führungskräfte mittels Fragebögen und Interviews, die Prozessanalyse erstellt daraus ein wissensorientiertes Prozessmodell. Die Lösungsdatenbank besteht aus einer Sammlung bewährter Methoden zum WM, die nach den Kriterien des GPO-WMModells (Geschäftsprozesse, WM-Kernaktivitäten, Gestaltungsfelder) klassifiziert sind. So lassen sich anhand des Prozessmodells schnell die notwendigen Maßnahmen ableiten.

Der Autor liefert mit diesem Buch ein integriertes Vorgehensmodell zur Optimierung wissensintensiver Geschäftsprozesse. Er verzichtet weitgehend auf die Generierung neuer und setzt auf die Komposition bewährter Modelle. So wird zur Modellierung der GP auf die Methode der integrierten Unternehmensmodellierung zurückgegriffen, und die Lösungsdatenbank besteht aus bewährten Methoden wie Yellow Pages, Foren etc. Besonderes Augenmerk legt der Autor auf die Einbeziehung der Mitarbeiter, die Darstellung von Fragebögen nimmt einen großen Teil des fünften Kapitels ein.

Fazit: Dieses Buch ist sicherlich eine interessante Lektüre für Führungspersonal, das sich mit der Optimierung des Wissensmanagements befasst und nach einer geeigneten Vorgehensweise sucht. Durch die Lösungsdatenbank kann schnell und unkompliziert die richtige Methode zur Lösung eines identifizierten Problems gefunden werden, vorausgesetzt, diese Datenbank wird laufend aktuell gehalten.

Christian Lütke Entrup, Siegen

Seibold, $H$.
IT-Risikomanagment
2. Auflage
ISBN 3-486-58009-4, Oldenbourg,
München 2006, 283 Seiten, $€ 39,80$

Dieses Werk ist der Versuch eines ganzheitlichen „Rundumschlags“ zur Sammlung aller möglichen Begriffe rund um IT-Risikomanagement, Informationstechnologie und der entsprechenden Normen. Selbst Schlagworte wie Corporate Governance, ITIL, Balance Scorecard und Risikowürfel (Risk Cube) sind zu finden. Besonders gut hat dem Rezensenten einleitend die grafische Übersicht mit der Abgrenzung des Themengebiets aus Sicht des Gesamtunternehmens gefallen.

In den ersten beiden Kapiteln wird sehr ausführlich auf Begriffsdefinitionen im Kontext Risikomanagement eingegangen. Etwas 